\title{
A Survey of Learning Needs about Oral Care for Intubated Patients among New Nursing Staff
}

\author{
Fumino Sugiyama ${ }^{\mathbb{D}}$, Tomoko Inoue \\ National College of Nursing, Tokyo, Japan \\ Email: sugiyamaf@adm.ncn.ac.jp, inout@adm.ncn.ac.jp
}

How to cite this paper: Sugiyama, F. and Inoue, T. (2020) A Survey of Learning Needs about Oral Care for Intubated $\mathrm{Pa}$ tients among New Nursing Staff. Open Journal of Nursing, 10, 462-471. https://doi.org/10.4236/ojn.2020.104032

Received: March 27, 2020

Accepted: April 25, 2020

Published: April 28, 2020

Copyright $\odot 2020$ by author(s) and Scientific Research Publishing Inc. This work is licensed under the Creative Commons Attribution International License (CC BY 4.0).

http://creativecommons.org/licenses/by/4.0/

(c) (i) Open Access

\begin{abstract}
Our aim was to understand the actual conditions of oral care for intubated patients by new nurses. This study was carried out from December 2017 to March 2018. We applied the theme of the dissipation of the dissociation between nursing skills learned in basic nursing education and nursing skills required in practice. A questionnaire survey was conducted at four facilities among new nursing staff who had acquired their nursing license in the past year. The questionnaire was composed of questions about their experiences of oral care for intubated patients, when they learned these skills, and what they wanted to learn. Answers were obtained from 118 new nurses. Learning about oral care was important for approximately $20 \%$ of the nurses, although it was not considered mandatory by all of the nurses. As infection control measures have revealed the acute needs of care for intubated patients, it is important for nursing students to learn about oral care and attitudes towards intubated patients, along with methods to make intubated patients more comfortable.
\end{abstract}

\section{Keywords}

New Nurses, Basic Nursing Education, Ventilator-Associated Pneumonia, Oral Care

\section{Introduction}

One important theme is dissipating the dissociation between the nursing skills required for clinical nursing practice and the nursing skills learned during basic nursing education. Thus, we aimed to understand the actual situation of oral care for intubated patients.

Although mechanical ventilation promotes the treatment of a causative disease through supporting a patient's ventilation or alterations of ventilation, it may cause serious complications. In particular, ventilator-associated pneumonia 
(VAP) is widely known to prolong hospital stays and increase medical care costs and mortality. As a measure to prevent VAP, strengthening the education of nurses has been shown to be related to a decrease in VAP incidence, mortality, and cost [1]; therefore, prevention measures are implemented according to the VAP bundle [2] [3] [4]. However, oral care is not included in the Japanese VAP bundle because the use of high-concentration $(0.12 \%-2 \%)$ chlorhexidine on the mucosa is prohibited in Japan due to the risk of anaphylaxis, although it is available in other countries, and thus methods for oral care of intubated patients vary [5].

In clinical practice, oral care is implemented for intubated patients more frequently than twice a day [6] [7]; however, the involvement of new nursing staff in this care and their learning needs have not been clarified. Intubated patients suffer from a dry mouth and growth of oral bacteria because they need to keep their mouth open; hence, they are usually administered a mouthwash/oral cavity moisturizer as part of their oral care. Nurses are required to have the skills to appropriately collect the liquids to prevent aspiration using a saliva ejector or suction tube, or by wiping the mouth, to prevent the liquids from invading to the bronchi via the intubation tube. While doing so, brushing or wiping is likely to cause a shift of the tracheal intubation tube, leading to damage to the airway mucosa, resulting in severe coughing. Therefore, two nursing staff are usually required for one oral care session for intubated patients; one supports the intubation tube, and the other cleans the oral cavity. For these reasons, new nurses are likely to need oral care skills. In addition, intubation can be an essential nursing skill acutely required at hub facilities for infection control or for securing the power supply in a time of disaster [8] [9]. On the other hand, it is not always a mandatory nursing skill for facilities that do not care for intubated patients. So, nurse learning needs depend on advancements in medical technology or specialization.

Based on the above, conducting a field survey to investigate the needs of oral care for intubated patients, leading to the development of educational materials that nursing students/new nurses can use anytime if necessary, is an urgent issue.

\section{Methods}

\subsection{The Operational Definition of Terms}

Intubated patients in this study were defined as patients who underwent tracheal intubation and wore an intubation tube. Oral care for intubated patients was defined as brushing of the oral cavity, adjusting the moisture environment in the oral cavity, or oral care associated with changes in the position of the intubation tube.

\subsection{Subjects and Survey Content}

Research cooperation was requested and accepted by the nursing directors of 
four institutions that have employed nursing students graduating from our college for adult nursing practicum. The institutions were selected from among the National Centers for Advanced and Specialized Medical Care hospitals accredited as advanced treatment hospitals. The directors of nursing distributed an online survey request to all new nurses (defined as having obtained their nurse's license less than one year ago). A total of 330 survey requests were distributed.

The questionnaire was designed by researchers based on previous studies, and two nurses in charge of new nurse training evaluated the validity of the instrument (shown in the appendix). The questionnaire asked about their experience with oral care for intubated patients, when they learned these skills, and what they wanted to learn.

\subsection{Data Collection and Analytical Approaches}

The survey period was between December 2017 and March 2018, and we sent the questionnaire with a reply envelope to the subjects through the nursing department of the targeted facilities. Their consent was established when they replied to the questionnaire and filled out the consent field. Descriptive statistics were used to calculate the experience and timing of oral care for intubated patients. Open questions related to learning needs were analyzed quantitatively and descriptively, integrating similar descriptions.

\subsection{Ethical Considerations}

The questionnaire was anonymous, and we paid attention to removing any personal information. Before the start of the survey, we obtained approval from the Ethical Review Board of the National Center for Global Health and Medicine (NCGM-G-002335-00).

\section{Results}

\subsection{Subject Outline}

Among the 120 total responders (response rate: $36.4 \%$ ), 118 agreed to participate in this study. There were 104 women (88.1\%), and their mean age was 23.9 years old. The most common educational facilities they had attended were nursing universities followed by nursing schools (Figure 1).

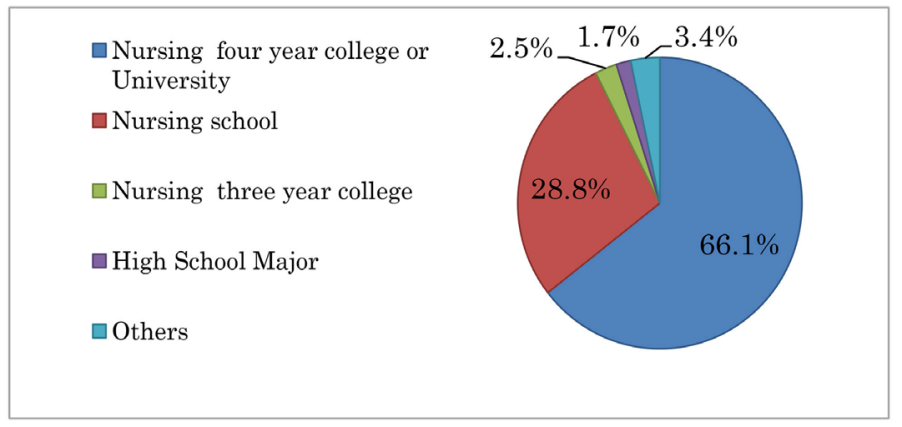

Figure 1. Training institutions $(\mathrm{n}=118)$. 


\subsection{Oral Care Experience for Intubated Patients}

Twenty-one (17.8\%) new nursing staff answered that they had oral care experience for intubated patients (Figure 2). For the question "In which work shift did you provide oral care" (multiple answers allowed), 15 subjects (75.0\%) answered "the night shift." For the question "Who do you work with when providing oral care" (multiple answers allowed), "a senior nurse" was the answer of 17 subjects (94.4\%).

\subsection{Learning Needs for Oral Care Approaches}

For learning needs $(\mathrm{n}=114), 52$ subjects $(45.6 \%)$ answered that they wanted to learn about oral care while they were students (Figure 3). For the question "What they wanted to learn when they were students $(\mathrm{n}=51), " 41$ subjects (80.4\%) answered "Lectures on how to provide oral care and things to pay attention to," 3 subjects (5.9\%) answered "Visiting a site of oral care," and 7 subjects (13.7\%) answered "training."

Answers for the question "When did you learn oral care for intubated patients" ( $\mathrm{n}=115$, multiple answers allowed) included "after being employed" 60 subjects (40.8\%) and "when they were students" 55 subjects (37.4\%). For the question "Do you have something to learn now as new nursing staff?" ( $\mathrm{n}=113$ ), 75 (66.4\%) subjects answered "Yes." The most common answer to "What do you want to learn now?" ( $\mathrm{n}=74)$, was "basic method" 51 subjects $(71.8 .1 \%)$.

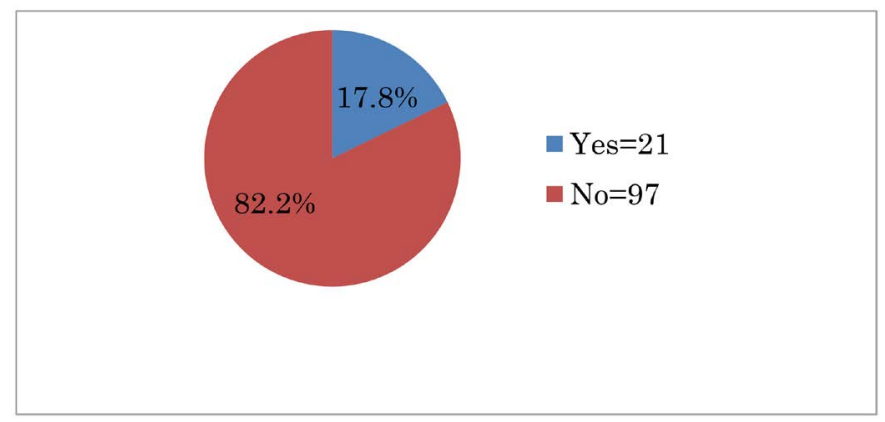

Figure 2. Experience of oral care for patients on mechanical ventilation $(\mathrm{n}=118)$.

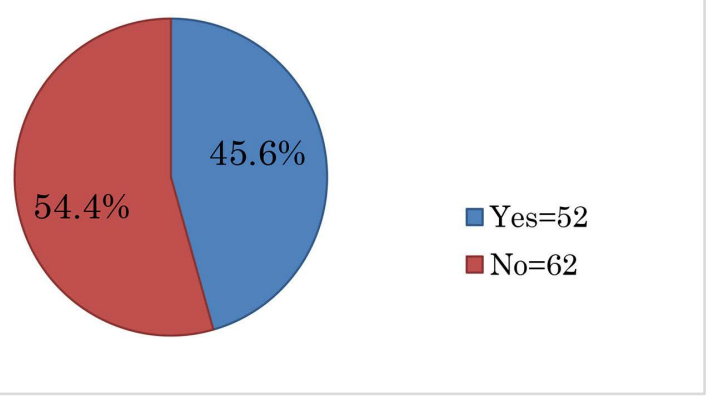

Figure 3. Things that they wanted to learn about care of patients on mechanical ventilators when they were students $(n=114)$. 
For the question "In providing oral care for intubated patients" ( $\mathrm{n}=20$, multiple answers allowed), 12 subjects (60.0\%) answered that they "had trouble." Answers for the question "What did you refer to while providing oral care for intubated patients" ( $\mathrm{n}=2$, multiple answers allowed) included "checklist used in the hospital ward" 11 subjects (55.0\%), "personally prepared list" 2 subjects (10.0\%), "not applicable" 4 subjects (20.0\%), and "other" 9 subjects (45.0\%).

Answers for the question "What role do you want to play in providing oral care for intubated patients" ( $\mathrm{n}=117$, multiple answers allowed) included "prevention of intubation-related pneumonia" 109 subjects (93.2\%), "moisten the dried oral cavity" 88 subjects (75.2\%), "Provide care so that patients can experience a fresh feeling" 84 subjects $(71.8 \%)$, "take good care of patients to free them from distress" 47 subjects (40.2\%), and "help team members such as senior staff or colleagues" 14 subjects (12.0\%) (Figure 4 ).

\section{Discussion}

\subsection{Learning Needs Related to Oral Care for Intubated Patients}

This survey's results showed that learning oral care was important for approximately $20 \%$ of new nurses. Oral care for intubated patients is not always necessary for all new nurses. However, previous studies showed that mechanical ventilators were used for tracheal intubation in $96 \%$ of 438 facilities, even in general hospital wards [10]. As oral care is also acutely required for infection control or disaster support, it is necessary to be prepared to provide educational opportunities combining e-learning and simulation education for nurses working at relevant facilities and for nursing students.

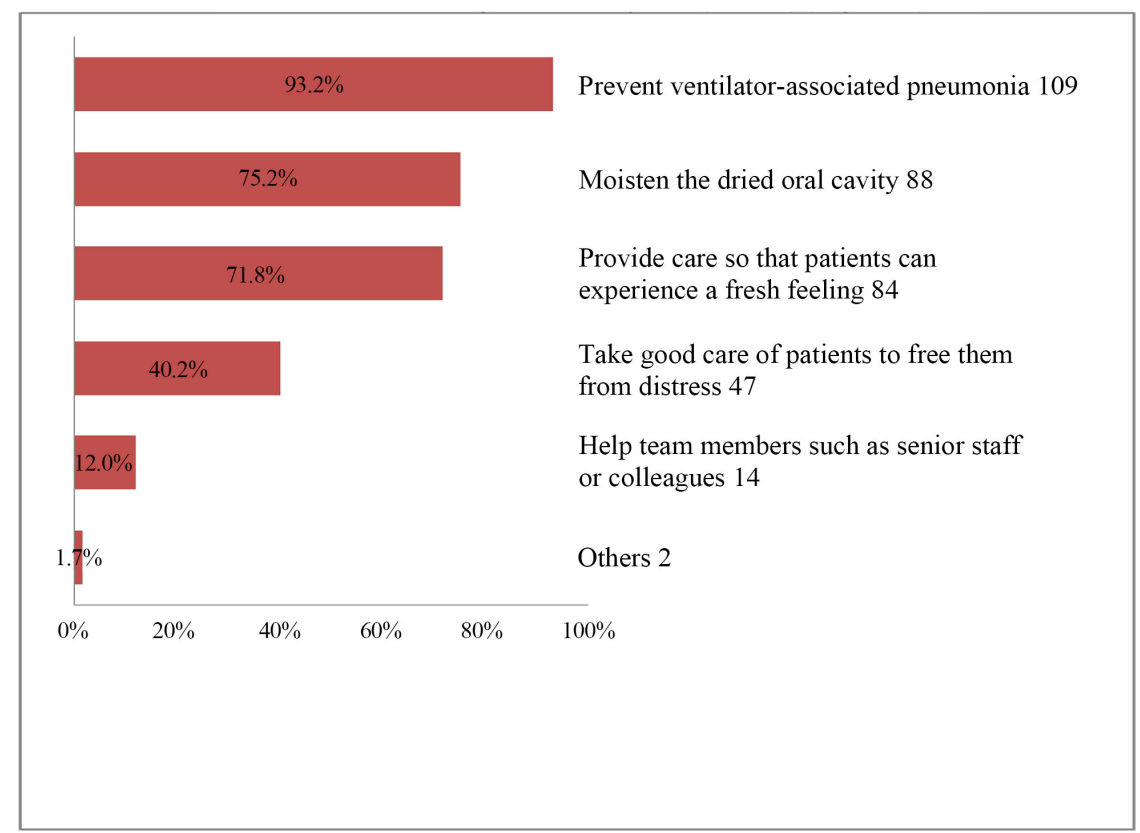

Figure 4. Things to refer to when providing care for patients on mechanical ventilators $(\mathrm{n}=117)$. 
New nursing staff with experience of oral care for intubated patients performed the care tasks under the supervision of senior nursing staff. Performing care tasks while learning from senior staff allows them to learn highly individualized condition assessments and care selection. This can be a strength in clinical practice. On the other hand, the results of this survey revealed a need for learning basic knowledge and implementing training about oral care for intubated patients. It was suggested that the most important factors at the level of intent influencing oral care for intubated patients are cognitive behavior control/attitude and knowledge about engaging in oral care [11]. Based on the above, integrating cognitive behavior control/attitude and knowledge, which help in understanding that oral care is a good practice, into nursing education as an educational subject, may help prevent VAP. Experiencing oral care for intubated patients in practical work is difficult because of the time necessary to take care of the patient and the complexity of the procedure. Therefore, if nurses who wish to work in the respiratory or intensive care areas shift their thoughts to learn oral care for intubated patients while they are a student via e-learning rather than on the job, the quantity and quality of clinical experience in VAP prevention may be enhanced.

\subsection{Comfort of Intubated Patients}

The results of this survey revealed that new nurses wanted to provide comfort to intubated patients through implementing oral care. "Prevention of VAP" was the primary answer to the question of the role they wanted to play in providing oral care for intubated patients, and it was followed by "moisten the dried oral cavity" and "provide care so that patients can experience a fresh feeling". This result showed that new nurses paid attention to comfort as well as prevention of VAP.

Katharine Kolcaba indicated three types of comfort [12]. One of them is "Relief," which means the condition in which pain is alleviated to a satisfactory level while managing the disease and receiving treatment procedures. The second one is "Ease", in which a patient can be calm without any anxiety. The third one is "Transcendence", which means the condition in which a patient can feel exaltation and confidence by inspiration and encouragement by others even though they still have pain or anxiety. If intubated patients can feel "Relief" or "Transcendence" through oral care, this may have important implications for patients.

Nurse-related literature reviews that were peer-reviewed and published in journals between 1960 and 2011 showed that oral care for intubated patients originally focused on patient comfort; however, it has been recently highlighted as a practical measure for control of VAP [13]. New nurses are able to assess pain in intubated patients because they understand that pain relief and comfort are necessary for the patient [14]. In an investigation of oral care in patients immediately after extubation, who were able to have a conversation, the patients said that their comfort was improved, and they felt relief. This result suggests 
that patients can feel comfortable even under intubation conditions if the protocol is unified [15]. Based on the above findings, a learning program including oral care for intubated patients that promotes an increase of comfort as well as reducing colonies of bacteria may help to dissipate the dissociation between the nursing skills required for clinical nursing practice and the nursing skills learned during nursing education.

\subsection{Limitations}

This study examined oral care for intubated patients by new nurses. This study was based on self-reported information, and reporting bias might have occurred. I tried to minimize this bias by providing multiple answer options to each question. Also, new nurses may have under-reported as to whether they had ever participated in oral care.

\section{Conclusion}

Oral care techniques for intubated patients are not always necessary for all new nurses. However, it is a necessary technique for some new nurses working in the respiratory area. It is important to gain knowledge and learn behavior about oral care for intubated patients and learn methods to make intubated patients feel comfortable while they are students to improve patient safety and to support new nurses.

\section{Acknowledgements}

We sincerely appreciate all of the new nurses who participated in this survey for taking time from their busy schedule to engage in nursing practical works and learning. This study was conducted with grant support from the Policy-based Medical Service Foundation 2019. A part of this study was published in "Iryo no Hiroba No. 58 Vol. 8 Pages 25-28 (2018.08)”.

\section{Conflicts of Interest}

The authors declare no conflicts of interest regarding the publication of this paper.

\section{References}

[1] Jansson, M., Kääriäinen, M. and Kyngäs, H. (2013) Effectiveness of Educational Programmes in Preventing Ventilator-Associated Pneumonia: A Systematic Review. Journal of Hospital Infection, 84, 206-214. https://doi.org/10.1016/j.jhin.2013.04.009

[2] Coffin, S.E., Klompas, M., Classen, D., et al. (2008) Strategies to Prevent Ventilator-Associated Pneumonia in Acute Care Hospitals. Infection Control \& Hospital Epidemiology, 29, S31-S40. https://doi.org/10.1086/591062

[3] The Japanese Society of Intensive Care Medicine, ICU Functional Assessment Committee (2010) Ventilator-Associated Pneumonia Prevention Bundle, 2010 Revised Edition. https://www.jsicm.org/pdf/2010VAP.pdf 
[4] Klompas, M., Branson, R., Eichenwald, E.C., et al. (2014) Strategies to Prevent Ventilator-Associated Pneumonia in Acute Care Hospitals: 2014 Update. Infection Control \& Hospital Epidemiology, 35, 915-936. https://doi.org/10.1086/677144

[5] Ryuichi, H. and Nobuaki, S. (2014) Is It Possible to Reduce the Incidence of Ventilator-Associated Pneumonia (VAP) to Zero? The Japanese Society of Intensive Care Medicine, 21, 9-16. https://doi.org/10.3918/jsicm.21.9

[6] Kazui, S. (2010) Hospital-Acquired Infection: V. Progresses of Diagnosis and Treatment Control of Patient with Special Pathological Conditions in Terms of Measures for Hospital-Acquired Infection. 1. Tracheal Intubation/Tracheostomy Cases. Internal Medicine, 97, 2724-2729.

[7] Yutaka, W., Genyuk, Y., Morio, T., et al. (2006) Oral Care of the Patients with Tracheal Intubation. Japanese Journal of Gerodontology, 20, 362-369.

[8] Health, Labour and Welfare Ministry (2020) Current List of Designated Medical Institutions for Specified Infectious Diseases (As of April 01, 2019).

https://www.mhlw.go.jp/bunya/kenkou/kekkaku-kansenshou15/02-02.html

[9] Bureau of Social Welfare and Public Health (2012) Tokyo Metropolitan Government. Support Guidance for Home Intubation Users in a Time of Disaster.

https://www.fukushihoken.metro.tokyo.lg.jp/smph/joho/soshiki/hoken/shippei/oshi $\underline{\text { rase/saigaijisiennsisinn.html }}$

[10] Tetsuya, M., Muneyasu, S. and Minoru, H. (2006) Field Survey of the Use of Intubation and Accessories. The Japanese Journal of Respiratory Care, 23, 92-97.

[11] Tanguay, A., LeMay, S., Reeves, I., et al. (2019) Factors Influencing Oral Care in Intubated Intensive Care Patients.

[12] Katharine, K. (2002) Comfort Theory and Practice: A Vision for Holistic Health Care and Research. Springer, Berlin.

[13] Dale, C., Angus, J.E., Sinuff, T., et al. (2013) Mouth Care for Orally Intubated Patients: A Critical Ethnographic Review of the Nursing Literature. Intensive and Critical Care Nursing, 29, 266-274. https://doi.org/10.1016/j.iccn.2012.09.003

[14] Ayasrah, S. (2016) Care-Related Pain in Critically Ill Mechanically Ventilated Patients. Anaesthesia and Intensive Care, 44, 458-465. https://doi.org/10.1177/0310057X1604400412

[15] Chipps, E.M., Carr, M., Kearney, R., et al. (2016) Outcomes of an Oral Care Protocol in Postmechanically Ventilated Patients. Worldviews on Evidence Based Nursing, 13, 102-111. https://doi.org/10.1111/wvn.12124 


\section{Appendix}

Questionnaire: Oral care for ventilator patients by new nursing staff:

- To be answered by those who have obtained a nurse's license less than a year ago.

- The experiences of those who have previously provided oral care to ventilator patients and those who have not are indispensable for developing teaching materials. We value your opinions. Thank you for your responses.

In this study, oral care for patients on ventilators refers to brushing the teeth of patients with tracheal intubation, adjusting the moisture in the oral cavity, and changing the fixed position of the intubation tube.

Q1 If you would like to fill out the Q1 survey, please select "agree" below.

Agree Disagree

Q2 Have you ever provided oral care to ventilator patients? Please select one.

Yes No

If you select "No," skip the following questions and resume taking the survey at Q9.

Q3 In what time period did you perform oral care for ventilator patients? Please select all that apply.

Day shift Night shift Other (please describe below):

Q4 How many people were involved in performing the oral care procedure? Please select all that apply.

One nurse Two nurses Other (please describe below):

Q5 Who did you work with while providing oral care for ventilator patients? Please select all that apply.

Peers Senior staff Other (please describe below):

Q6 Did you refer to anything when performing oral care for ventilator patients? Please select all that apply.

No reference Checklist used in the ward Checklist prepared by individuals Other (please describe below):

Q7 Have you had any difficulties with oral care for ventilator patients? Please select one corresponding number and circle it.

Yes No If you select "Yes," please describe what you were having trouble with:

Q8 Have you ever planned an oral care session for ventilator patients?

Yes No If you select "Yes," please describe your oral care planning experience:

Q9 When did you learn about oral care for ventilator patients? Please select all that apply.

I had a lecture when I was a student.

Exercises when I was a student.

Observed in practical training as a student.

Implemented in practical training as a student.

There was training after I got a job. 
I learned from senior staff in the ward.

I watched senior staff in the ward.

Other (please describe below):

Q10 What role do you want to play in oral care procedures for ventilator patients? Please select all that apply.

Moisturizing dry mouths.

Prevent ventilator-associated pneumonia.

Help patients feel refreshed.

Helping fellows who are taking care of the oral cavity together, such as seniors and colleagues.

Ensure the patient is without pain.

Other (please describe below):

Q11 Have you ever wanted to learn about oral care for ventilator patients as a student?

Yes No If you select "Yes," please state what you wanted to have learned:

Q12 Do you want to learn about oral care for ventilator patients right now?

Yes No If you select "Yes," please state what you want to learn:

Q13 Please enter your age

Q14 Please select your gender.

Male Female

Q15 Which of the following training institutions is applicable to you?

Nursing three years college Nursing four years college or university Nursing school High school major

Other (please describe below):

Q16 If you have any additional comments on anything related to oral care for ventilator patients, please let us know.

Q17 Please let us know if you have any comments about this study.

That's it for the questionnaire.

Thank you for your cooperation. 